\title{
OPTICAL LEPTONS
}

\author{
LUBOMIR M. KOVACHEV
}

Received 2 January 2003

\begin{abstract}
We obtain optical vortices with classical orbital momentum $\ell=1$ and spin $j= \pm 1 / 2$ as exact solutions of a system of nonlinear Maxwell equations (NMEs). Two kinds of Kerr-type media, namely, those with and without linear dispersion of the electric and the magnet susceptibility, are investigated. The electric and magnetic fields are represented as sums of circular and linear components. This allows us to reduce the NME to a set of nonlinear Dirac equations (NDEs). The vortex solutions in the case of media with dispersion admit finite energy, while the solutions in case of media without dispersion admit infinite energy. The amplitude equations are obtained from equations of nonstationary optical and magnetic response (dispersion). This includes also the optical pulses with time duration of order of and less than the time of relaxation of the media (femtosecond pulses). The possible generalization of NME to a higher number of optical components and a higher number of $\ell$ and $j$ is discussed.
\end{abstract}

2000 Mathematics Subject Classification: 35L60, 45G15, 74J30.

1. Introduction. Nonlinear wave processes appeared in different sections of the contemporary physics: nonlinear optics, plasma physics, hydrodynamics, and nonlinear field theory. Specific problems and methods of investigation exist in all of these sections. However, it is possible to find a lot of regularities, connected with the evolution of the nonlinear localized waves, in spite of their different physical interpretation. In the last five decades, there has been considerable interest in the nonlinear generalizations of the quantum field equations $[12,15,18]$ and in the possibility of obtaining exact stationary solitary solutions of the field equations $[3,11]$. As a rule, the nonlinearity has been introduced in an ad hoc fashion in the Klein-Gordon equation and also for all four spinor components of the Dirac equations. For the usual case of a cubic nonlinearity, exact localized solutions are not found. Our present work, reported in this paper, shows that the optical analogy of nonlinear Dirac equations (NDEs) leads to a nonlinear part in only the first coupled equation of NDEs. This result allows to solve the NDE by separation of variables and to obtain solutions representing optical vortices with classical momenta one and spin one half. The initial investigation of optical vortices began with a scalar theory, based on the well-known $2 \mathrm{D}+1$ paraxial nonlinear Schrödinger equation (NSE) [19, 22]. The existence of optical vortices was predicted in the self-focusing regime, but as it was shown in many papers, the solutions obtained are modulationally unstable. In spite of this, various interactions (attraction, repulsion, and fusion) have been observed. The scalar paraxial approximation is valid for slowly varying amplitudes of the electrical field in weakly nonlinear media. As it was pointed out in $[10,17]$, this theory is not valid for very intense narrow pulses. The first generalization 
of the scalar paraxial theory of optical vortices is based on the investigation of the so-called spatiotemporal scalar evolution equations [4, 9, 21]. For all cited theories, no exact solutions have been found, but numerical and energy momentum techniques are used. The existence of exact stable vortex solutions of these types of nonlinear equations was finally discovered with the vector generalization of the 3D +1 NSE [2]. It has also been shown numerically that these vortices are stable at distances comparable to those where localized solutions of the one-component scalar equation are self-focusing rapidly. All of these exact vortices are a combination of linearly polarized components and have $\operatorname{spin} \ell=1$. To extend the theory to vortices with $\operatorname{spin} j=1 / 2$, we return to an analogy between the Maxwell and Dirac equations. As it is shown in [8], this analogy is possible only if the electrical and magnet components are represented as a sum of linear and circularly polarized components.

2. Maxwell's equations: nonstationary linear and nonlinear polarization (dispersion). Consider Maxwell's equations in the next two cases:

(1) a source-free medium with nonstationary linear and nonlinear electric polarization and nonstationary magnetic polarization (the case with dispersion),

(2) a source-free medium with stationary linear and nonlinear electric polarization and stationary magnetic polarization (the case without dispersion).

For these cases, Maxwell's equations can be written as

$$
\begin{gathered}
\nabla \times \vec{E}=-\frac{1}{c} \frac{\partial \vec{B}}{\partial t}, \quad \nabla \times \vec{H}=\frac{1}{c} \frac{\partial \vec{D}}{\partial t}, \quad \nabla \cdot \vec{D}=0, \\
\nabla \cdot \vec{B}=\nabla \cdot \vec{H}=0, \quad \vec{D}=\vec{P}]^{\operatorname{lin}}+4 \pi \vec{P}_{n \ell},
\end{gathered}
$$

with the corresponding linear magnetic polarization $[5,16]$

$$
\vec{B}=\vec{H}+4 \pi \vec{M}_{\text {lin }},
$$

where $\vec{E}$ and $\vec{H}$ are the electric and magnetic intensity fields, $\vec{D}$ and $\vec{B}$ are the electric and magnetic induction fields, $\vec{P}^{\text {lin }}$ and $\vec{P}_{n \ell}$ are the linear and nonlinear polarization of the medium, respectively, and $\vec{M}_{\text {lin }}$ is the linear magnetic polarization. The magnetic polarization (magnetization) $\vec{M}_{\text {lin }}$ is written as the product of the linear magnetic susceptibility $\eta^{(1)}$ and the magnetic field $\vec{H}$. The nonstationary linear electric polarization can be written as

$$
\begin{aligned}
\vec{P}^{\text {lin }} & =\int_{-\infty}^{t}\left(\delta(t-\tau)+4 \pi X^{(1)}(t-\tau)\right) \vec{E}(\tau, x, y, z) d \tau \\
& =\int_{-\infty}^{t} \varepsilon_{0}(t-\tau) \vec{E}(\tau, x, y, z) d \tau,
\end{aligned}
$$

where $\chi^{(1)}$ and $\varepsilon_{0}$ are the linear electric susceptibility and the dielectric constant, respectively. A similar expression describes the dependence of $\vec{B}$ on $\vec{H}$ in the case of 
nonstationary linear magnetic polarization [14]:

$$
\begin{aligned}
\vec{B} & =\int_{-\infty}^{t}\left(\delta(t-\tau)+4 \pi \eta^{(1)}(t-\tau)\right) \vec{H}(\tau, x, y, z) d \tau \\
& =\int_{-\infty}^{t} \mu_{0}(t-\tau) \vec{H}(\tau, x, y, z) d \tau,
\end{aligned}
$$

where $\eta^{(1)}$ and $\mu_{0}$ are the linear magnetic susceptibility and the magnetic permeability, respectively. The magnetic susceptibility of the main part of the dielectrics ranges from $10^{-6}$ to $10^{-4}$ and usually decreases with the increase of the frequency. In the following, we will study such media with nonstationary cubic nonlinear polarization, where the nonlinear polarization in the case of one carrying frequency can be expressed as

$$
\begin{aligned}
\vec{P}_{\mathrm{nlin}}^{(3)}=\frac{3}{4} \int_{-\infty}^{t} \int_{-\infty}^{t} \int_{-\infty}^{t} & x^{(3)}\left(t-\tau_{1}, t-\tau_{2}, t-\tau_{3}\right) \\
& \times \vec{E}\left(\tau_{1}, x, y, z\right) \vec{E}^{*}\left(\tau_{2}, x, y, z\right) \vec{E}\left(\tau_{3}, x, y, z\right) d \tau_{1} d \tau_{2} d \tau_{3} .
\end{aligned}
$$

The causality requires the next conditions on the response functions:

$$
\varepsilon(t-\tau)=0, \quad x^{(3)}\left(t-\tau_{1}, t-\tau_{2}, t-\tau_{3}\right)=0, \quad t-\tau<0, t-\tau_{i}<0, i=1,2,3 .
$$

That is why we can prolong the upper integral boundary to infinity to use the standard Fourier presentation [16]:

$$
\begin{aligned}
& \int_{-\infty}^{t} \varepsilon_{0}(\tau-t) \exp (i \omega \tau) d \tau=\int_{-\infty}^{+\infty} \varepsilon_{0}(\tau-t) \exp (i \omega \tau) d \tau, \\
& \int_{-\infty}^{t} \int_{-\infty}^{t} \int_{-\infty}^{t} x^{(3)}\left(t-\tau_{1}, t-\tau_{2}, t-\tau_{3}\right) d \tau_{1} d \tau_{2} d \tau_{3} \\
& \quad=\int_{-\infty}^{+\infty} \int_{-\infty}^{+\infty} \int_{-\infty}^{+\infty} x^{(3)}\left(t-\tau_{1}, t-\tau_{2}, t-\tau_{3}\right) d \tau_{1} d \tau_{2} d \tau_{3} .
\end{aligned}
$$

The spectral presentation of linear optical susceptibility $\hat{\varepsilon}_{0}(\omega)$ is connected to the nonstationary optical response function by the next Fourier transform:

$$
\hat{\varepsilon}_{0}(\omega) \exp (i \omega t)=\int_{-\infty}^{+\infty} \varepsilon_{0}(t-\tau) \exp (i \omega \tau) d \tau .
$$

Similar expressions for the spectral presentation of the nonstationary magnetic response $\hat{\mu}_{0}(\omega)$,

$$
\hat{\mu}_{0}(\omega) \exp (-i \omega t)=\int_{-\infty}^{+\infty} \mu_{0}(t-\tau) \exp (-i \omega \tau) d \tau,
$$

and nonlinear optical susceptibility $\chi^{(3)}$,

$$
\begin{aligned}
\hat{\chi}^{(3)} \exp (-i \omega t)=\int_{-\infty}^{+\infty} \int_{-\infty}^{+\infty} \int_{-\infty}^{+\infty} & X^{(3)}\left(t-\tau_{1}, t-\tau_{2}, t-\tau_{3}\right) \\
& \times \exp \left(i\left(\omega\left(\tau_{1}+\tau_{2}+\tau_{3}\right)\right)\right) d \tau_{1} d \tau_{2} d \tau_{3},
\end{aligned}
$$


can be written. It is important to point out here the remark of Akhmanov et al. in [1], that such nonstationary representation applied to slowly varying amplitudes of electrical and magnet fields is valid as well when the optical pulse duration of the pulses $t_{0}$ is greater than the characteristic response time of the media $\tau_{0}\left(t_{0} \gg \tau_{0}\right)$, as when the time duration of the pulses is less than or equal to the time response of the media $\left(t_{0} \leq \tau_{0}\right)$. We will discuss this possibility in the process of deriving the amplitude equations.

3. Derivation of the amplitude equations. In this section, we derive the slowly varying amplitude approximation in the standard way, as it was done in [13, 16]. For the case of Maxwell's equations with linear and nonlinear dispersion (2.1) and (2.2), we define the electric and magnetic fields amplitudes by the following relations:

$$
\begin{aligned}
\vec{E}(x, y, z, t) & =\vec{A}(x, y, z, t) \exp \left(i\left(\omega_{0} t-g(x, y, z)\right)\right), \\
\vec{H}(x, y, z, t) & =\vec{C}(x, y, z, t) \exp \left(-i\left(\omega_{0} t-q(x, y, z)\right)\right),
\end{aligned}
$$

where $\vec{A}, \vec{C}, \omega_{0}, g$, and $q$ are the amplitudes of the electric and magnetic fields, the optical frequency, and the real spatial phase functions, respectively. In the case of monochromatic and quasimonochromatic fields, the Stokes parameters can be constructed from transverse components of the wave field [5, 7]. This leads to two-component vector fields in a plane, transverse to the direction of propagation. For electromagnetic fields with spectral bandwidth (our case), the two-dimensional coherency tensor cannot be used and the Stokes parameters cannot be found directly. As it was shown by Carozzi et al. in [7], using a high order of symmetry (SU(3)), in this case, six independent Stokes parameters can be found. This corresponds to a three-component vector field. Here, this case is investigated. The increase of the spectral bandwidth of the vector wave also increases the depolarization term (component, normal to the standard Stokes coherent polarization plane).

We use the Fourier representation of the response functions (2.8), (2.9), and (2.10) and of the amplitude functions $\vec{A}$ (and $\vec{C}$ ) to obtain next expressions for the first derivatives in time of the linear polarization, nonlinear polarization, and magnetic induction fields:

$$
\begin{aligned}
\frac{1}{c} \frac{\partial \vec{P} \operatorname{lin}(x, y, z, t)}{\partial t}= & i \exp \left(i\left(\omega_{0} t-g(x, y, z)\right)\right) \\
& \times \int_{-\infty}^{+\infty} \frac{\omega \hat{\varepsilon}_{0}(\omega)}{c} \vec{A}\left(x, y, z, \omega-\omega_{0}\right) \exp \left(i\left(\omega-\omega_{0}\right) t\right) d \omega, \\
\frac{4 \pi}{c} \frac{\partial \vec{P} \operatorname{lin}(x, y, z, t)}{\partial t}= & i \exp \left(i\left(\omega_{0} t-g(x, y, z)\right)\right) \\
& \times \int_{-\infty}^{+\infty} \frac{3 \pi \omega \hat{\chi}^{(3)}(\omega)}{c}\left|\vec{A}\left(x, y, z, \omega-\omega_{0}\right)\right|^{2} \vec{A}\left(x, y, z, \omega-\omega_{0}\right) \\
& \quad \times \exp \left(i\left(\omega-\omega_{0}\right) t\right) d \omega,
\end{aligned}
$$




$$
\begin{aligned}
-\frac{1}{c} \frac{\partial \vec{B}(x, y, z, t)}{\partial t}= & i \exp \left(i\left(\omega_{0} t-q(x, y, z)\right)\right) \\
& \times \int_{-\infty}^{+\infty} \frac{\omega \hat{\mu}_{0}(\omega)}{c} \vec{C}\left(x, y, z, \omega-\omega_{0}\right) \exp \left(-i\left(\omega-\omega_{0}\right) t\right) d \omega .
\end{aligned}
$$

At this point, we restrict the spectrum of the amplitude of electrical and magnet fields by writing the wave vectors $k_{1, \text { nlin,2 }}$ in a Taylor series:

$$
\begin{aligned}
k_{1}(\omega) & =\frac{\omega \hat{\varepsilon}_{0}(\omega)}{c}=k_{1}^{0}\left(\omega_{0}\right)+\frac{\partial\left(k_{1}\left(\omega_{0}\right)\right)}{\partial \omega}\left(\omega-\omega_{0}\right)+\frac{1}{2} \frac{\partial^{2}\left(k_{1}\left(\omega_{0}\right)\right)}{\partial \omega^{2}}\left(\omega-\omega_{0}\right)^{2}+\cdots \\
& =k_{1}^{0}\left(\omega_{0}\right)+\frac{1}{v_{1}}\left(\omega-\omega_{0}\right)+\frac{1}{2} k_{1}^{\prime \prime}\left(\omega-\omega_{0}\right)^{2}+\cdots \\
k_{\text {nlin }}(\omega) & =\frac{3 \pi \omega \hat{\chi}^{(3)}(\omega)}{c}=k_{\text {nlin }}^{0}\left(\omega_{0}\right)+\frac{\partial\left(k_{\text {nlin }}\left(\omega_{0}\right)\right)}{\partial \omega}\left(\omega-\omega_{0}\right)+\cdots \\
& =k_{\text {nlin }}^{0}\left(\omega_{0}\right)+\frac{1}{v_{\text {nlin }}}\left(\omega-\omega_{0}\right)+\cdots, \\
k_{2}(\omega) & =\frac{\omega \hat{\mu}_{0}(\omega)}{c}=k_{2}^{0}\left(\omega_{0}\right)+\frac{\partial\left(k_{2}\left(\omega_{0}\right)\right)}{\partial \omega}\left(\omega-\omega_{0}\right)+\frac{1}{2} \frac{\partial^{2}\left(k_{2}\left(\omega_{0}\right)\right)}{\partial \omega^{2}}\left(\omega-\omega_{0}\right)^{2}+\cdots \\
& =k_{2}^{0}\left(\omega_{0}\right)+\frac{1}{v_{2}}\left(\omega-\omega_{0}\right)+\frac{1}{2} k_{2}^{\prime \prime}\left(\omega-\omega_{0}\right)^{2}+\cdots,
\end{aligned}
$$

where $v_{i}$ and $k_{i}^{\prime \prime}, i=1$, nlin, 2, have dimensions of group velocity, and nonlinear addition to the group velocity and dispersion, respectively. The nonlinear wave vector is expressed as

$$
k_{\mathrm{nlin}}^{0}=\frac{3 \pi \omega_{0} \hat{\chi}^{(3)}\left(\omega_{0}\right)}{c}=\frac{\omega_{0} \hat{\varepsilon}_{0}}{c} \frac{3 \pi \hat{\chi}^{(3)}\left(\omega_{0}\right)}{\hat{\varepsilon}_{0}}=k_{1} n_{2},
$$

where

$$
n_{2}\left(\omega_{0}\right)=\frac{3 \pi \hat{\chi}^{(3)}\left(\omega_{0}\right)}{\hat{\varepsilon}_{0}}
$$

is the nonlinear refractive index. It is important to note here that we do not use any approximation of the response function. There is only one requirement of the spectral presentations (2.10), (2.8), and (2.9) of the response functions: to admit first- and second-order derivatives in respect to frequency (to be smooth functions). The restriction is only in respect of the relation between the main frequency $\omega_{0}$ and time duration of the envelope functions $t_{0}$ determinate from the relations (3.5), (3.6), and (3.7) (conditions for slowly varying amplitudes). Putting (3.5) in (3.2), (3.7) in (3.4), and (3.6) in (3.3), and keeping in mind the expressions for time derivatives of the spectral presentation of the amplitude functions, the first derivatives (3.2), (3.3), and (3.4) are presented in 
the form

$$
\begin{aligned}
\frac{1}{c} \frac{\partial \vec{P} \operatorname{lin}(x, y, z, t)}{\partial t}= & \left(i k_{1}^{0} \vec{A}+\frac{1}{v_{1}} \frac{\partial \vec{A}}{\partial t}-\frac{i k_{1}^{\prime \prime}}{2} \frac{\partial^{2} \vec{A}}{\partial t^{2}}\right) \\
& \times \exp \left(i\left(\omega_{0} t-g(x, y, z)\right)\right), \\
\frac{4 \pi}{c} \frac{\partial \vec{P} \operatorname{nlin}(x, y, z, t)}{\partial t}= & \left(i k_{1}^{0} n_{2}|\vec{A}|^{2} \vec{A}+\frac{1}{v_{\text {nlin }}} \frac{\partial|\vec{A}|^{2} \vec{A}}{\partial t}\right) \\
& \times \exp \left(i\left(\omega_{0} t-g(x, y, z)\right)\right), \\
-\frac{1}{c} \frac{\partial \vec{B}(x, y, z, t)}{\partial t}= & \left(i k_{2}^{0} \vec{C}+\frac{1}{v_{2}} \frac{\partial \vec{C}}{\partial t}-\frac{i k_{2}^{\prime \prime}}{2} \frac{\partial^{2} \vec{C}}{\partial t^{2}}\right) \\
& \times \exp \left(i\left(\omega_{0} t-q(x, y, z)\right)\right) .
\end{aligned}
$$

Finally, from Maxwell's equations (2.1) and (2.2), using (3.10) and using the fact that $\nabla$. $\vec{D} \approx \nabla \cdot \vec{E} \approx 0$ [2], we obtain the next system of vector amplitude NMEs:

$$
\begin{gathered}
\nabla \times \vec{A}=i k_{2}^{0} \vec{C}-\frac{1}{v_{2}} \frac{\partial \vec{C}}{\partial t}-\frac{i k_{2}^{\prime \prime}}{2} \frac{\partial^{2} \vec{C}}{\partial t^{2}}, \\
\nabla \times \vec{C}=i k_{1}^{0} \vec{A}+\frac{1}{v_{1}} \frac{\partial \vec{A}}{\partial t}-\frac{i k_{1}^{\prime \prime}}{2} \frac{\partial^{2} \vec{A}}{\partial t^{2}}+i k_{1}^{0} n_{2}\left(\vec{A} \cdot \vec{A}^{*}\right) \vec{A}+\left(\frac{n_{2}}{v_{1}}+k_{1} \frac{\partial n_{2}}{\partial \omega}\right) \frac{\partial\left(\vec{A} \cdot \vec{A}^{*}\right) \vec{A}}{\partial t}, \\
\nabla \cdot \vec{A}=0, \quad \nabla \cdot \vec{C}=0,
\end{gathered}
$$

if the gradient of the spatial phase functions $g$ and $q$ satisfy the relations

$$
\nabla g \times \vec{A}=0, \quad \nabla q \times \vec{C}=0
$$

The phase functions whichever satisfy (3.12) are determinate in Section 10.

We investigate the case when our vector fields are presented as a sum of circular and linear polarizing components:

$$
\vec{A}=\vec{A}_{\text {lin }}+\vec{A}_{\text {cir }}, \quad \vec{C}=\vec{C}_{\text {lin }}+\vec{C}_{\text {cir }}
$$

The nonlinear polarization admits different nonlinear refractive indexes in the case of linear and circular polarization [6] $\left(n_{2}^{\text {lin }} \neq n_{2}^{\text {cir }}\right)$. We will include this difference in our rescaled equations, defining the rescaled dependant variables

$$
\vec{A}=A_{0}^{\operatorname{lin}} \vec{A}_{\text {lin }}^{\prime}+A_{0}^{\text {cir }} \vec{A}_{\text {cir }}^{\prime}, \quad \vec{C}=C_{0}\left(\vec{C}_{\text {lin }}^{\prime}+\vec{C}_{\text {cir }}^{\prime}\right), \quad\left(A_{0}^{\operatorname{lin}}\right)^{2}=\frac{n_{2}^{\text {cir }}}{n_{2}^{\operatorname{lin}}}\left(A_{0}^{\mathrm{cir}}\right)^{2},
$$

and the independent variables

$$
x=r_{0} x^{\prime}, \quad y=r_{0} y^{\prime}, \quad z=r_{0} z^{\prime}, \quad t=t_{0} t^{\prime} .
$$


In addition, the next constants are determinate:

$$
\begin{gathered}
\alpha_{i}=k_{i}^{0} r_{0}, \quad \beta_{i}=\frac{k_{i}^{\prime \prime} r_{0}}{2 t_{0}^{2}}, \quad \gamma_{1}=r_{0} k_{1} n_{2}^{\text {cir }}\left|A_{0}\right|^{2}, \\
\gamma_{2}=n_{2}^{\text {cir }}\left|A_{0}\right|^{2}, \quad \gamma_{3}=\frac{v_{1} n_{2}^{\text {cir }}\left|A_{0}\right|^{2}}{c}, \\
\delta=\frac{v_{1}}{v_{2}} \leq 1, \quad v_{1} \approx \frac{r_{0}}{t_{0}}, \quad i=1,2 .
\end{gathered}
$$

The NMEs (3.11) in rescaled variables are transformed into the following (the primes have been omitted for clarity):

$$
\begin{gathered}
\nabla \times \vec{A}=i \alpha_{2} \vec{C}-\delta \frac{\partial \vec{C}}{\partial t}-i \beta_{2} \frac{\partial^{2} \vec{C}}{\partial t^{2}} \\
\nabla \times \vec{C}=i \alpha_{1} \vec{A}+\frac{\partial \vec{A}}{\partial t}-i \beta_{1} \frac{\partial^{2} \vec{A}}{\partial t^{2}}+i \gamma_{1}\left(\vec{A} \cdot \vec{A}^{*}\right) \vec{A}+\left(\gamma_{2}+\gamma_{3}\right) \frac{\partial\left(\left(\vec{A} \cdot \vec{A}^{*}\right) \vec{A}\right)}{\partial t}, \\
\nabla \cdot \vec{A}=0, \quad \nabla \cdot \vec{C}=0 .
\end{gathered}
$$

We consider the case of slowly varying amplitudes approximation when the nonlinear constant is $\gamma_{1}=1$. Then the constants in front of the last term on the right-hand side are $\gamma_{2} \approx \gamma_{3} \approx 10^{-2}-10^{-3}$, while the constants $\alpha_{i}$ have typical values $\alpha \approx 10^{2}-10^{3}$ $\left(\alpha_{i} \sim r_{0} k_{i}\right)$ and the dispersion constants $\beta_{i}$ have very small values $\beta_{1} \sim 10^{-5}-10^{-6}$ for picosecond and subpicosecond pulses in the transparency region of nonlinear optical media. Neglecting the small dispersion terms $\beta_{i} \ll 1$ and the last nonlinear term for which $\gamma_{2} \approx \gamma_{3} \ll 1$, the NMEs system (3.17) can be rewritten as

$$
\begin{gathered}
\nabla \times \vec{A}=i \alpha_{2} \vec{C}-\delta \frac{\partial \vec{C}}{\partial t}, \\
\nabla \times \vec{C}=i \alpha_{1} \vec{A}+\frac{\partial \vec{A}}{\partial t}+i \gamma_{1}\left(\vec{A} \cdot \vec{A}^{*}\right) \vec{A} \\
\nabla \cdot \vec{A}=0, \quad \nabla \cdot \vec{C}=0 .
\end{gathered}
$$

4. Dirac representation of NME. To solve the NMEs (3.18), we apply the method of separation of variables. The slowly varying amplitude vectors of the electric field $\vec{A}$ and the magnetic field $\vec{C}$ are represented as

$$
\begin{aligned}
& \vec{A}(x, y, z, t)=\vec{F}(x, y, z) \exp (i \Delta \alpha t), \\
& \vec{C}(x, y, z, t)=\vec{G}(x, y, z) \exp (i \Delta \alpha t) .
\end{aligned}
$$

Substituting these forms into the NMEs (3.18), we obtain

$$
\begin{gathered}
\nabla \times \vec{F}=-i \nu_{2} \vec{G}, \\
\nabla \times \vec{G}=i v_{1} \vec{F}+i \gamma_{1}\left(\vec{F} \cdot \vec{F}^{*}\right) \vec{F}, \\
\nabla \cdot \vec{F}=0, \quad \nabla \cdot \vec{G}=0,
\end{gathered}
$$

where $\nu_{1}=\alpha_{1}+\Delta \alpha, \nu_{2}=\delta \Delta \alpha-\alpha_{2}>0$. In a Cartesian coordinate system, the vector equations (4.2) are reduced to a scalar system of eight nonlinear wave equations. When 
the electric and magnetic fields are represented as a sum of a linear polarization component and a circular polarized one, it is possible to reduce (4.2) to a system of four nonlinear equations. Substituting [8]

$$
\begin{aligned}
& \psi_{1}=i F_{l}=i F_{z}, \\
& \psi_{2}=F_{c}=i F_{x}-F_{y}, \\
& \psi_{3}=G_{l}=-G_{z}, \\
& \psi_{4}=G_{c}=-G_{x}-i G_{y}
\end{aligned}
$$

into the nonlinear system (4.2), we obtain a stationary system of NDEs:

$$
\begin{gathered}
\left(\frac{\partial}{\partial x}-i \frac{\partial}{\partial y}\right) \Psi_{4}+\frac{\partial}{\partial z} \Psi_{3}=-i\left(\nu_{1}+\gamma_{1} \sum_{i=1}^{2}\left|\Psi_{i}\right|^{2}\right) \Psi_{1}, \\
\left(\frac{\partial}{\partial x}+i \frac{\partial}{\partial y}\right) \Psi_{3}-\frac{\partial}{\partial z} \Psi_{4}=-i\left(\nu_{1}+\gamma_{1} \sum_{i=1}^{2}\left|\Psi_{i}\right|^{2}\right) \Psi_{2}, \\
\left(\frac{\partial}{\partial x}-i \frac{\partial}{\partial y}\right) \Psi_{2}+\frac{\partial}{\partial z} \Psi_{1}=-i \nu_{2} \Psi_{3}, \\
\left(\frac{\partial}{\partial x}+i \frac{\partial}{\partial y}\right) \Psi_{1}-\frac{\partial}{\partial z} \Psi_{2}=-i \nu_{2} \Psi_{4} .
\end{gathered}
$$

This substitution allows to reduce the system of eight equations (4.2) to a system of four scalar complex equations (4.4). System (4.4) is the optical analog of the NDEs. Note that the optical NDEs are significantly different from the NDEs in the field theory. The nonlinear part appears only in the first two coupled equations of the system.

5. Vortex solutions with orbital momentum $l=1$ and $\operatorname{spin} j=1 / 2$. The symmetries of the NDEs (4.4) are used to obtain exact vortex solutions. The NDEs (4.4) have both spherical and spinor symmetries only in the case where the nonlinear part does not manifest the angular dependence on the radial variable $\sum_{i=1}^{2}\left|\Psi_{i}(r, \theta, \varphi)\right|^{2}=F(r)$. This type of solutions can be found using the following technique. Using Pauli matrices, we write the NDEs system (4.4) as

$$
\begin{gathered}
(\vec{\sigma} \cdot \vec{P}) \phi=-i\left(\nu_{1}+\gamma_{1} \sum_{i=1}^{2}\left|\eta_{i}\right|^{2}\right) \eta, \\
(\vec{\sigma} \cdot \vec{P}) \eta=-i v_{2} \phi
\end{gathered}
$$

where

$$
\vec{\sigma}=\left[\left(\begin{array}{ll}
0 & 1 \\
1 & 0
\end{array}\right),\left(\begin{array}{cc}
0 & -i \\
i & 0
\end{array}\right)\left(\begin{array}{cc}
1 & 0 \\
0 & -1
\end{array}\right)\right]
$$

are the Pauli matrices, $\vec{P}=(\partial / \partial x, \partial / \partial y, \partial / \partial z)$ is the differential operator, and

$$
\eta=\left(\begin{array}{l}
\Psi_{1} \\
\Psi_{2}
\end{array}\right), \quad \phi=\left(\begin{array}{l}
\Psi_{3} \\
\Psi_{4}
\end{array}\right)
$$


are the corresponding spinors. After substituting (5.2) into (5.1), we obtain

$$
(\vec{\sigma} \cdot \vec{P})(\vec{\sigma} \cdot \vec{P}) \eta=-v_{2}\left(v_{1}+\gamma_{1} \sum_{i=1}^{2}\left|\eta_{i}\right|^{2}\right) \eta
$$

When there is no external electric or magnetic field, the operator on the left-hand side of (5.5) is the Laplacian operator $\Delta$,

$$
P^{2}=(\vec{\sigma} \cdot \vec{P})(\vec{\sigma} \cdot \vec{P})=\Delta .
$$

From (5.5) and (5.6) we obtain

$$
v_{2} v_{1} \eta+v_{2} \gamma_{1} \sum_{i=1}^{2}\left|\eta_{i}\right|^{2} \eta+\Delta \eta=0
$$

In the case of spherical representation of the spinor equations (5.5), there are two possibilities, $l=0$ and $l=1$, that will permit only a radial dependence of the nonlinear part:

$$
\sum_{i=1}^{2}\left|\eta_{i}(r, \theta, \varphi)\right|^{2}=F(r)
$$

For the $l=0$ case, equations (5.7) are transformed to equations with radial parts:

$$
v_{2} v_{1} \eta+v_{2} \gamma_{1} \sum_{i=1}^{2}\left|\eta_{i}\right|^{2} \eta+\frac{\partial^{2} \eta}{\partial r^{2}}+\frac{2}{r} \frac{\partial \eta}{\partial r}=0
$$

The scalar variant of these equations has been investigated in many papers, but exact localized solutions have not been found. In the case $l=1$, we look for spinors in the next two forms:

$$
\eta=\left(\begin{array}{c}
\tilde{\eta}(r) \cos (\theta) \\
\tilde{\eta}(r) \sin (\theta) \exp (i \varphi)
\end{array}\right), \quad \eta=\left(\begin{array}{c}
\tilde{\eta}(r) \sin (\theta) \exp (-i \varphi) \\
-\tilde{\eta}(r) \cos (\theta)
\end{array}\right) .
$$

As it will be seen later, these correspond to two opposite directions of the intrinsic orbital momentum $j= \pm 1 / 2$ (opposite charges). After substituting solutions (5.10) into (5.7), the following equation describing the radial dependence is obtained:

$$
\nu_{2} v_{1} \tilde{\eta}+v_{2} \gamma_{1}|\tilde{\eta}|^{2} \tilde{\eta}+\frac{\partial^{2} \tilde{\eta}}{\partial r^{2}}+\frac{2}{r} \frac{\partial \tilde{\eta}}{\partial r}-\frac{2}{r^{2}} \tilde{\eta}=0
$$

The angular parts are the standard spherical harmonics with $l=1$. Using the fact that

$$
\left|\frac{\exp \left(i \sqrt{\nu_{1} \nu_{2}} r\right)}{r}\right|=\frac{\sqrt{\nu_{1} \nu_{2}}}{r}
$$

it is straightforward to show that the radial part (5.11) admits exact vortex de Broglie soliton solutions [3] in the form

$$
\tilde{\eta}(r)=\frac{\sqrt{2} \exp \left(i \sqrt{\nu_{1} \nu_{2}} r\right)}{r} \quad \text { or } \quad \tilde{\eta}(r)=\frac{\sqrt{2}}{i} \frac{\sin \left(\sqrt{\nu_{1} \nu_{2}} r\right)}{r}
$$


if $v_{2} \gamma_{1}=1$. The complete solutions for these two cases are written as

$$
\begin{aligned}
& \eta=\left(\begin{array}{c}
\frac{\sqrt{2} \exp \left(i \sqrt{\nu_{1} \nu_{2}} r\right)}{r} \cos (\theta) \\
\frac{\sqrt{2}}{i} \frac{\exp \left(i \sqrt{\nu_{1} \nu_{2}} r\right)}{r} \sin (\theta) \exp (i \varphi)
\end{array}\right) \\
& \phi=\left(\begin{array}{c}
\frac{\sqrt{2}}{i \nu_{2}}\left(\frac{i \sqrt{\nu_{1} \nu_{2}} \exp \left(i \sqrt{\nu_{1} \nu_{2}} r\right)}{r}+\frac{\exp i\left(\sqrt{\nu_{1} \nu_{2}} r\right)}{r^{2}}\right) \\
0
\end{array}\right) ; \\
& \eta=\left(\begin{array}{c}
\frac{\sqrt{2}}{i} \frac{\exp \left(i \sqrt{\nu_{1} \nu_{2}} r\right)}{r} \sin (\theta) \exp -(i \varphi) \\
\frac{-\sqrt{2} \exp \left(i \sqrt{\nu_{1} \nu_{2}} r\right)}{r} \cos (\theta)
\end{array}\right), \\
& \phi=\left(\begin{array}{c}
0 \\
\frac{-\sqrt{2}}{i \nu_{2}}\left(\frac{i \sqrt{\nu_{1} \nu_{2}} \exp \left(i \sqrt{\nu_{1} \nu_{2}} r\right)}{r}+\frac{\exp i\left(\sqrt{\nu_{1} \nu_{2}} r\right)}{r^{2}}\right)
\end{array}\right) .
\end{aligned}
$$

There is another more direct way for separating the variables of the spinor equations (5.1) and (5.2). To illustrate this, we represent the NDEs (4.4) in spherical variables:

$$
\begin{aligned}
& \exp (-i \varphi)\left(\sin \theta \frac{\partial}{\partial r}+\frac{\cos \theta}{r} \frac{\partial}{\partial \theta}-\frac{i}{r \sin \theta} \frac{\partial}{\partial \varphi}\right) \Psi_{4}+\left(\cos \theta \frac{\partial}{\partial r}-\frac{\sin \theta}{r} \frac{\partial}{\partial \theta}\right) \Psi_{3} \\
& =-i\left(v_{1}+\gamma_{1} \sum_{i=1}^{2}\left|\Psi_{i}\right|^{2}\right) \Psi_{1}, \\
& \exp (i \varphi)\left(\sin \theta \frac{\partial}{\partial r}+\frac{\cos \theta}{r} \frac{\partial}{\partial \theta}+\frac{i}{r \sin \theta} \frac{\partial}{\partial \varphi}\right) \Psi_{3}-\left(\cos \theta \frac{\partial}{\partial r}-\frac{\sin \theta}{r} \frac{\partial}{\partial \theta}\right) \Psi_{4} \\
& =-i\left(v_{1}+\gamma_{1} \sum_{i=1}^{2}\left|\Psi_{i}\right|^{2}\right) \Psi_{2}, \\
& \exp (-i \varphi)\left(\sin \theta \frac{\partial}{\partial r}+\frac{\cos \theta}{r} \frac{\partial}{\partial \theta}-\frac{i}{r \sin \theta} \frac{\partial}{\partial \varphi}\right) \Psi_{2}+\left(\cos \theta \frac{\partial}{\partial r}-\frac{\sin \theta}{r} \frac{\partial}{\partial \theta}\right) \Psi_{1} \\
& =-i v_{2} \Psi_{3}, \\
& \exp (i \varphi)\left(\sin \theta \frac{\partial}{\partial r}+\frac{\cos \theta}{r} \frac{\partial}{\partial \theta}+\frac{i}{r \sin \theta} \frac{\partial}{\partial \varphi}\right) \Psi_{1}-\left(\cos \theta \frac{\partial}{\partial r}-\frac{\sin \theta}{r} \frac{\partial}{\partial \theta}\right) \Psi_{2} \\
& =-i v_{2} \Psi_{4} .
\end{aligned}
$$

We make the following two ansatzes for solutions to the system of nonlinear equations (5.18):

$$
\begin{gathered}
\Psi_{1}=a(r) \cos (\theta), \quad \Psi_{2}=a(r) \sin (\theta) e^{i \varphi}, \quad \Psi_{3}=-i b(r), \quad \Psi_{4}=0 ; \\
\Psi_{1}=a(r) \sin (\theta) e^{-i \varphi}, \quad \Psi_{2}=-a(r) \cos (\theta), \quad \Psi_{3}=0, \quad \Psi_{4}=-i b(r) .
\end{gathered}
$$


Put (5.19) or (5.20) in (5.18). We separate the variables. The following system of equations describing the radial dependence of the amplitudes is obtained:

$$
\begin{gathered}
\frac{\partial a(r)}{\partial r}+\frac{2}{r} a(r)=-v_{2} b(r) \\
\frac{\partial b(r)}{\partial r}=v_{1} a(r)+\gamma_{1}|a(r)|^{2} a(r) .
\end{gathered}
$$

In the system of (5.21), the nonlinearity appears only in the radial part, while for the angular part, we have standard spherical spinors with spin $j= \pm 1$. Solving (5.21), we straightforwardly show that when $v_{2} \gamma=1$, these equations admit the localized de Broglie solitons of (5.14).

6. Hamiltonian representation of the NDE. First integrals for vortex solutions with spin $j= \pm 1 / 2$. It is not difficult to show that for the NDEs system of (4.4), a Hamiltonian of the form

$$
H=(\vec{\sigma} \cdot \vec{P})+\gamma \sum_{i=1}^{2}\left|\Psi_{i}\right|^{2}
$$

can be written. Using this, (4.4) can be rewritten in the form

$$
H \Psi=\varepsilon \Psi,
$$

where $\gamma=\left(\gamma_{1}, \gamma_{1}, 0,0\right)$ and $\varepsilon=\left(-i \nu_{1},-i \nu_{1},-i \nu_{2},-i \nu_{2}\right)$ is the energy operator. Here, we investigate the case where the nonlinear part of the equation is represented as a number of spinors with a scalar sum that depends only on the radial component:

$$
\sum_{i=1}^{2}\left|\Psi_{i}(r, \theta, \varphi)\right|^{2}=F(r) .
$$

We also introduce here the well-known orbital momentum operator $\vec{L}$, intrinsic orbital (spin) momentum $\vec{S}$, and the full momentum $\vec{J}$, as well as

$$
\vec{L}=\vec{r} \times \vec{P}, \quad \frac{1}{2} \vec{S}=\frac{1}{2}\left(\begin{array}{cc}
\vec{\sigma} & 0 \\
0 & \vec{\sigma}
\end{array}\right), \quad \vec{J}=\vec{L}+\frac{1}{2} \vec{S}
$$

It is straightforward to show that the Hamiltonian (6.1) of (6.2) commutes with the operators $\vec{J}^{2}$ and $J_{z}$ (the $z$-projections must be $x$ or $y$ ). Using these symmetries and the condition that the nonlinearity is of Kerr type, we can solve the NDE (6.2) by a separation-of-variables technique. We look for solutions in the form

$$
\Psi_{1}=a(r) \Omega_{j l M}, \quad \Psi_{2}=a(r) \Omega_{j l M}, \quad \Psi_{3}=i b(r) \Omega_{j l^{\prime} M}, \quad \Psi_{4}=i b(r) \Omega_{j l^{\prime} M},
$$

where $\Omega_{j l m}$ is the spherical spinor, $l+l^{\prime}=1$, and $a(r)$ and $b(r)$ are arbitrary radial functions. Using the symmetries of (6.2) and the fact that the nonlinear parts depend 
on $r$, we separate variables and obtain the following system of equations for the radial part:

$$
\begin{gathered}
\frac{\partial a(r)}{\partial r}+\frac{1+\chi}{r} a(r)=-v_{2} b(r), \\
\frac{\partial b(r)}{\partial r}+\frac{1-\chi}{r} b(r)=v_{1} a(r)+\gamma|a(r)|^{2} a(r),
\end{gathered}
$$

where

$$
\chi=l(l+1)-j(j+1)-\frac{1}{4}
$$

Excluding $b(r)$ from system (6.6), we obtain the next equation for $a(r)$ :

$$
v_{1} v_{2} a(r)+\frac{\partial^{2} a}{\partial r^{2}}+\frac{2}{r} \frac{\partial a}{\partial r}-\frac{(1+\chi) \chi}{r^{2}} a+v_{2} \gamma|a|^{2} a=0
$$

Formally, this equation admits exact "de Broglie" soliton solutions for an arbitrary number of $\chi$. But, as we remember, our solutions are limited by condition (6.3) with the nonlinear part depending only on the radial components. Condition (6.3) for a number $x \geq 1$ can be fulfilled also for a higher number of fields on different frequencies. This case includes also the parametric processes. The case of one carrying frequencies corresponds to localized solutions with $\chi=1$, angular components $l=1$, and $j= \pm 1 / 2$. In this case, system (6.6) becomes

$$
\begin{gathered}
\frac{\partial a(r)}{\partial r}+\frac{2}{r} a(r)=-v_{2} b(r), \\
\frac{\partial b(r)}{\partial r}=v_{1} a(r)+\gamma|a(r)|^{2} a(r) .
\end{gathered}
$$

As shown above, this system has exact radial solutions of the form (5.13), (5.14), and (5.15).

7. Experimental conditions. There are some differences between the nonlinear conditions for localized solutions of the vector nonlinear Schrodinger equation (VNLS) and the localized conditions for the nonlinear Maxwell equations (NMEs). The nonlinear parameter for the VNLS is written [2] as

$$
\gamma_{\mathrm{vnls}}=k_{0}^{2} r_{0}^{2} n_{2}\left|A_{0}\right|^{2}=1
$$

For localized solutions in optical region, the constant $\alpha^{2}$ ranges between

$$
\alpha^{2}=k_{0}^{2} r_{0}^{2} \approx 10^{4}-10^{6}
$$

which corresponds to a required nonlinear refractive index change of the order of

$$
n_{2}\left|A_{0}\right|^{2} \approx 10^{-4}-10^{-6} \text {. }
$$


On the other hand, the nonlinear condition for localized solutions of the normalized NDEs (3.18) is

$$
\gamma_{\mathrm{NDE}}=v_{2} \gamma_{1} \approx\left(\delta \Delta \alpha-\alpha_{2}\right) k_{1} r_{0} n_{2}\left|A_{0}\right|^{2}=1
$$

For the typical value of the constant $v_{2} \approx 1$, the next required nonlinear refractive index change appears:

$$
n_{2}\left|A_{0}\right|^{2} \approx 10^{-2}-10^{-3}
$$

Another difference between these two cases is that the solutions of the VNLS are comprised of linearly polarized components and that the dispersion of the nonlinear medium plays an important role. This leads to the fact that vortex solutions of the VNLS may be observed only in special dispersion regions of nonlinear media. The vortices of the NDEs are a combination of linear and circular polarization components, do not have this marked dependence on the dispersion, and may be observed in the transparency region of nonlinear media.

8. Vortex solutions in nonlinear Kerr-type media without dispersion. The case without linear dispersion of the electrical and magnetic susceptibility will correspond to $\chi^{(1)}=$ const and $\eta^{(1)}=$ const. We suppose now that the amplitude functions do not depend on time and will look for 3D +1 monochromatic electric and magnet fields of the kind

$$
\begin{aligned}
\vec{E}(x, y, z, t) & =\vec{M}(x, y, z) \exp \left(i \omega_{0} t\right), \\
\vec{H}(x, y, z, t) & =\vec{N}(x, y, z) \exp \left(-i \omega_{0} t\right),
\end{aligned}
$$

where $\vec{M}, \vec{N}$, and $\omega_{0}$ are the amplitudes of the electric and magnetic fields and the optical frequency, respectively.

Substituting the relations (8.1), one obtains the next amplitude equations in the case of dispersionless media:

$$
\begin{gathered}
\nabla \times \vec{M}=i \alpha_{2} \vec{N}, \\
\nabla \times \vec{N}=i \alpha_{1} \vec{M}+\gamma\left(\vec{M} \cdot \vec{M}^{*}\right) \vec{M}, \\
\nabla \cdot \vec{M}=0, \quad \nabla \cdot \vec{N}=0 .
\end{gathered}
$$

Again, the electric and magnetic fields are represented as a sum of a linear polarization component and a circular polarized one. Substituting

$$
\begin{aligned}
& \psi_{1}=i M_{l}=i M_{z}, \\
& \psi_{2}=M_{c}=i M_{x}-M_{y}, \\
& \psi_{3}=N_{l}=-i N_{z}, \\
& \psi_{4}=N_{c}=-N_{x}-i N_{y}
\end{aligned}
$$


into the nonlinear system (8.2), we obtain the same kind of stationary system of NDEs as (4.4),

$$
\begin{gathered}
\left(\frac{\partial}{\partial x}-i \frac{\partial}{\partial y}\right) \Psi_{4}+\frac{\partial}{\partial z} \Psi_{3}=-i\left(\kappa_{1}+\zeta \sum_{i=1}^{2}\left|\Psi_{i}\right|^{2}\right) \Psi_{1}, \\
\left(\frac{\partial}{\partial x}+i \frac{\partial}{\partial y}\right) \Psi_{3}-\frac{\partial}{\partial z} \Psi_{4}=-i\left(\kappa_{1}+\zeta \sum_{i=1}^{2}\left|\Psi_{i}\right|^{2}\right) \Psi_{2}, \\
\left(\frac{\partial}{\partial x}-i \frac{\partial}{\partial y}\right) \Psi_{2}+\frac{\partial}{\partial z} \Psi_{1}=-i \kappa_{2} \Psi_{3}, \\
\left(\frac{\partial}{\partial x}+i \frac{\partial}{\partial y}\right) \Psi_{1}-\frac{\partial}{\partial z} \Psi_{2}=-i \kappa_{2} \Psi_{4},
\end{gathered}
$$

but with different coefficients $\kappa_{1}=\omega_{0} \varepsilon_{0} / c, \kappa_{2}=\omega_{0} \mu_{0} / c$, and $\zeta=4 \pi \omega_{0} \chi(3) / c$. Naturally, the NDEs (8.4) admit the same kind of solutions (5.14) and (5.15).

9. Conditions for finiteness of the energy of the vortex solutions. In this section, we will investigate the localized energy in both cases, with and without linear dispersion. These correspond to two kinds of optical vortices, with and without spectral bandwidth. In dielectric media without dispersion, the expression for the linear part of energy density is

$$
W_{\text {lin }}=\frac{1}{8 \pi}\left(\varepsilon|\vec{E}|^{2}+\mu|\vec{H}|^{2}\right),
$$

where $\varepsilon$ and $\mu$ are constants. Substituting the vortex solutions (5.14) and (5.15) in (9.1) and integrating over 3D space, one obtains that quasimonochromatic vortices in dielectric media without dispersion admit infinite energy. Now, we come to the case of slowly varying amplitude approximation and calculation of energy of the vortex solutions of media with linear electric and magnet dispersion. To prove the finiteness of energy of the vortex solutions (5.14) and (5.15), we start with the equations for averaged-in-time balance of energy density of electrical and magnet fields [14]:

$$
\left\langle\frac{\partial W}{\partial t}\right\rangle=\frac{1}{16 \pi}\left(\vec{E} \cdot \frac{\partial \vec{D}^{*}}{\partial t}+\vec{E}^{*} \cdot \frac{\partial \vec{D}}{\partial t}+\vec{B} \cdot \frac{\partial \vec{B}^{*}}{\partial t}+\vec{B}^{*} \cdot \frac{\partial \vec{B}}{\partial t}\right),
$$

where $\vec{D}=\vec{P}_{\text {lin }}+4 \pi \vec{P}_{\text {nlin }}$ is a sum of the linear induction and the nonlinear induction of the electrical field. The calculations of the averaged energy of the optical waves in dispersive media are worked out considering the first order of slowly varying amplitude approximation of electrical induction (the same as in the NDEs). The result comes to the old result of Brillouin (1921) for energy density of electrical field:

$$
\left\langle W_{\text {lin }}\right\rangle=\frac{1}{8 \pi}\left(\frac{\partial\left(\omega \hat{\varepsilon}_{0}\right)}{\partial \omega}|\vec{A}|^{2}+\frac{\partial\left(\omega \hat{\mu}_{0}\right)}{\partial \omega}|\vec{C}|^{2}\right) .
$$

The condition of electric constant

$$
\hat{\varepsilon}_{0}>0, \quad \frac{\partial\left(\omega \hat{\varepsilon}_{0}\right)}{\partial \omega}>0
$$


is fulfilled in most of the dielectrics. The conditions of the magnetic constant can be present in two cases. In the first one is

$$
\hat{\mu}_{0}>0, \quad \frac{\partial\left(\omega \hat{\mu}_{0}\right)}{\partial \omega}>0
$$

This condition corresponds to the case when there are not any magnet relaxation processes included. To include these processes is possible only for ultrashort optical pulses with time duration of dimension of local structure of one paramagnetic medium. The real part of magnet susceptibility in this case is a symmetric function in respect to the paramagnetic resonance, and the derivative in respect to the frequency is always negative,

$$
\hat{\mu}_{0}>0, \quad \frac{\partial \hat{\mu}_{0}(\omega)}{\partial \omega}<0
$$

Under appropriate conditions, this leads to

$$
\hat{\mu}_{0}>0, \quad \frac{\partial\left(\omega \hat{\mu}_{0}(\omega)\right)}{\partial \omega}<0
$$

We can find spectral region and dispersion parameters when the next condition is satisfied:

$$
\frac{\partial\left(\omega \hat{\mu}_{0}\right)}{\partial \omega}=-\frac{\partial\left(\omega \hat{\varepsilon}_{0}\right)}{\partial \omega}
$$

Thus, the linear (infinity) part of energy density is zero. The nonlinear part of averaged energy density is expressed in [20] and for the vortex solutions (5.14) and (5.15) becomes

$$
\left\langle W_{\text {nlin }}\right\rangle=n_{2}|\Psi|^{2} \mathfrak{R}\left(\Psi^{2}\right)+\omega_{0} \frac{\partial n_{2}}{\partial \omega_{0}}|\Psi|^{2} \mathfrak{R}\left(\Psi^{2}\right) .
$$

These results give the conditions for the finiteness of energy of the vortices. Integrating $W_{\text {nlin }}$ in the 3D space, we obtain a finite value proportional to the main frequency $\omega_{0}$.

10. Spatial phase functions, Poynting vector, and flow of energy. The kind of the phase functions which satisfy (3.12) is determinate for vortex solutions (5.19) with spin $j=1 / 2$. Using again the relations between the spinors of NDEs and the amplitude functions (4.3), we have

$$
\begin{array}{ccc}
F_{x}=\frac{\psi_{2}-\psi_{2}^{*}}{2 i}, & F_{y}=\frac{\psi_{2}+\psi_{2}^{*}}{2}, & F_{z}=\frac{\psi_{1}-\psi_{1}^{*}}{2 i}, \\
G_{x}=-\frac{\psi_{4}+\psi_{4}^{*}}{2}, & G_{y}=-\frac{\psi_{4}-\psi_{4}^{*}}{2 i}, & G_{z}=-\frac{\psi_{3}-\psi_{3}^{*}}{2 i} .
\end{array}
$$


Substituting the solutions (5.19) with spin $j=1 / 2$ in (10.1) for the real radial solutions of kind $\sin (\alpha r) / r$, we obtain

$$
\begin{gathered}
F_{x}=-\frac{\sin (\alpha r)}{r} \frac{x}{r}, \quad F_{y}=-\frac{\sin (\alpha r)}{r} \frac{y}{r}, \quad F_{z}=-\frac{\sin (\alpha r)}{r} \frac{z}{r}, \\
G_{x}=0, \quad G_{y}=0, \quad G_{z}=\frac{\alpha \cos (\alpha r)}{r}+\frac{\sin (\alpha r)}{r^{2}} .
\end{gathered}
$$

We rewrite again the conditions for the spatial phase functions

$$
\nabla g \times \vec{F}=0, \quad \nabla q \times \vec{G}=0 .
$$

These relations for solutions of kind (10.2) and (10.3) are satisfied only when

$$
\begin{gathered}
g=k_{0} r \quad \text { or } \quad g=k_{0} f(r), \\
q=k_{0} r, \quad q=k_{0} z, \quad \text { or } \quad q=f(z),
\end{gathered}
$$

where $k_{0}$ is the carrying wave number. The spatial phase functions of kind $g=k_{0} r$ and $q=k_{0} z$ correspond to spectral limited pulses which satisfy additional relations

$$
\triangle k \triangle r=\text { const. }
$$

The spatial phase functions of kind $g=k_{0} f(r)$ and $q=k_{0} f(z)$ correspond to phase modulated pulses and for them the relations (10.6) are not satisfied. The Poynting vector can be expressed by the amplitude functions of the electrical and magnet fields:

$$
\begin{aligned}
\vec{S} & =\vec{E}(x, y, z, t) \times \vec{H}(x, y, z, t) \\
& =\exp (i(W(t)-K(r))) \vec{F}(x, y, z, t) \times \vec{G}(x, y, z, t),
\end{aligned}
$$

where $W$ and $K$ are scalar phase functions. Substituting the solutions with $\operatorname{spin} j=1 / 2$ in the above expression, we find that

$$
\vec{S}=\exp (i(W(t)-K(r)))\left(-a(r) b(r) \frac{y}{r} ; a(r) b(r) \frac{x}{r} ; 0\right) .
$$

We see that the Poynting vector $\vec{S}$ is a one-circulation vector for solutions with spin $j=1 / 2$ and its divergency is zero,

$$
\nabla \cdot \vec{S}=0
$$

The relation (10.9) determines that the energy flow through arbitrary closed surface around our vortex solutions with spin $j=1 / 2$ is zero. The relation (10.8) shows that flow of energy of our solutions circulates in $x, y$ plane. Now, we can generalize the above results for solutions with spin $j=1 / 2$ : the vortex solutions with spin $j=1 / 2$ without external fields are immovable and electromagnetic energy oscillates in $x, y$ plane. The electrical field oscillates spherically, in " $r$ " direction, while the magnet field oscillates in $z$ direction. In the same way was calculated the Poynting vector for solutions with spin $j=-1 / 2$. For them, we obtain that $\nabla \cdot \vec{S} \neq 0$ and we expect that they are not stable. One exact investigation of stability request investigation is also the perturbation 


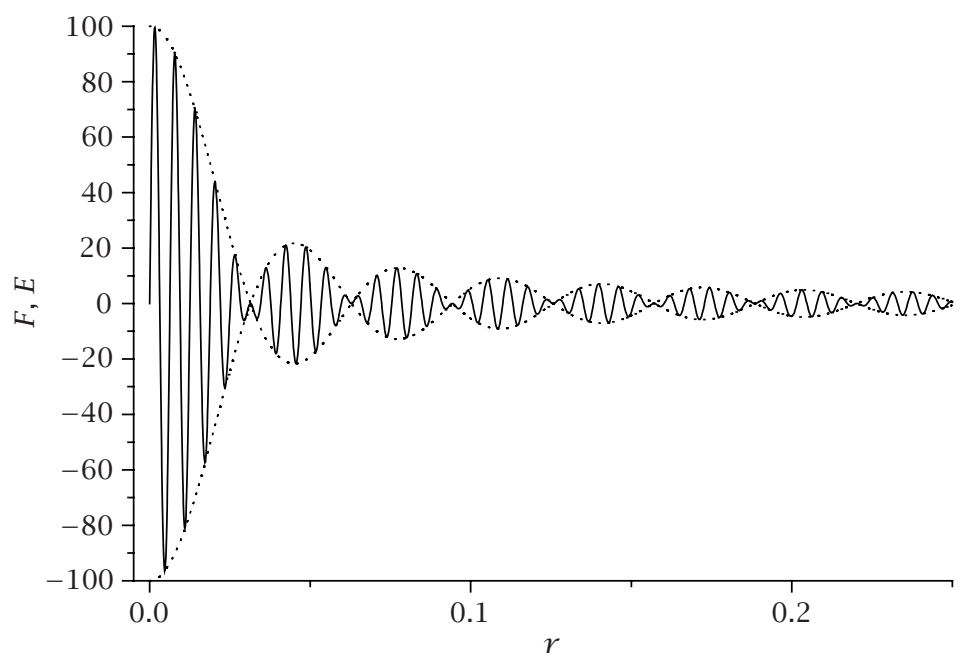

FIGURE 10.1. Radial distribution of the envelop solutions $F(r)$ of NDEs (dotted line) and the corresponding real electrical field $E(r)$ (solid line). The real field tends to zero in the origin.

of the Poynting theorem and will be discussed in a next paper. We will discuss here more accurately the connection between the spatial shapes of the envelop functions for electrical (10.2) and magnetic (10.3) fields with the spatial shapes of the real (sin) part of the electric and magnetic fields. Investigating the shape of the envelope function of magnetic field (10.3), it is straightforward to show that the solutions tend to zero in infinity and are indeterminate in the origin.

It is a well-known fact that the envelop solutions (10.2) and (10.3) are only imaginary functions around the real electrical and magnetic fields which are presented in this case by

$$
E_{i}=F_{i} \sin \left(k_{0} r\right), \quad H_{z}=G_{z} \sin \left(k_{0} r\right),
$$

where $k_{0}$ is the normalized carrying wave number, $i=x, y, z$, and

$$
k_{0} \gg \alpha
$$

Concerning the real fields (10.10) in the origin and infinity, it can be seen that they admit finite value in the origin and tend to zero at infinity. Practically, the envelop approximation gives unique possibility to study envelopes, which are indefinite in one point or on a line. This is shown on Figures 10.1 and 10.2 where the shapes of radial part of electric envelopes $F_{i}$ and the envelop of magnetic field $G_{z}$ (dotted line) are plotted together with the real electrical $E_{i}$ and magnetic field $H_{z}$ (solid line). In Figure 10.1 , the radial part of the envelop solutions of NDEs is presented by

$$
F(r)=\frac{\sin \left(\left(v_{1} v_{1}\right)^{1 / 2} \cdot r\right)}{r}
$$




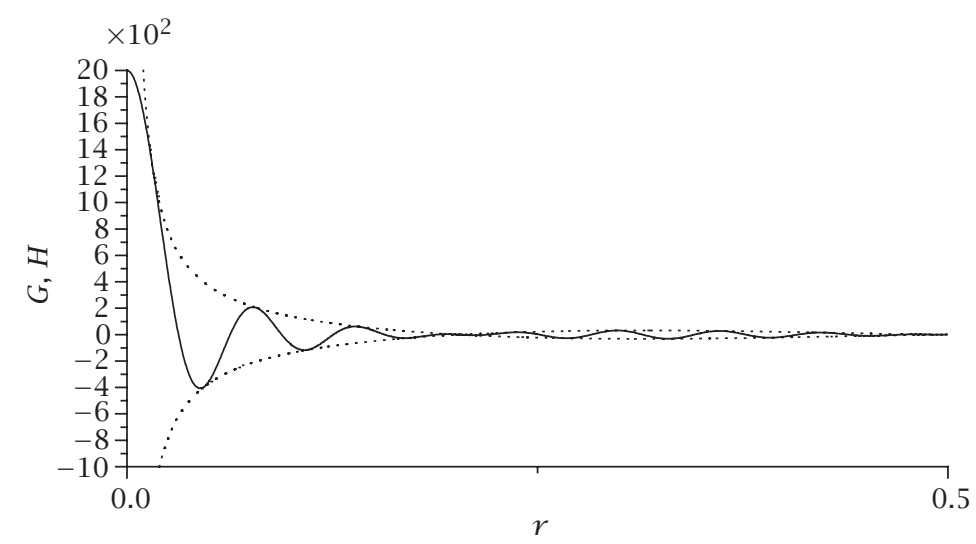

FIGURE 10.2. Radial distribution of the envelop solutions $G(r)$ of NDEs (dotted line) and the corresponding real magnetic field $H(r)$ (solid line). The real field tends to finite value in the origin.

and the electrical field is presented by

$$
\begin{gathered}
E(r)=F(r) \cdot \sin \left(k_{0} \cdot r\right), \\
k_{0}=1000, \quad v_{1} v_{2}=100 .
\end{gathered}
$$

As for Figure 10.2, the envelop solutions of NDEs for the magnetic field are presented by

$$
G(r)=\frac{\left(v_{1} v_{2}\right)^{1 / 2} \cos \left(\left(v_{1} v_{2}\right)^{1 / 2} r\right)}{r}+\frac{\sin \left(\left(v_{1} v_{2}\right)^{1 / 2} r\right)}{r},
$$

while the magnetic field is presented by

$$
H(r)=G(r)^{*} \sin \left(k_{0} r\right)
$$

It is clearly seen that the amplitude functions are only imaginary functions around the real field with $2 \pi$-accuracy. The corresponding real fields are well determined and have no singularities at the origin and at infinity it tends to zero.

If we multiply the radial functions of the electrical field $E_{i}$ with the corresponding angular spherical harmonics, we see that the complete solution for the electrical fields is determined in the origin also. We use the following normalized wave vectors $\alpha=100$, $k_{0}=1000$ for electrical field and $k_{0}=100, k_{1}=50$ for the magnetic field, and in this way we keep the correlation between these and the real wave vectors.

Such kinds of problems, with indefiniteness of the functions in the origin, arise also in the field theory. When solutions of the linear Dirac and Schrödinger equations are products of the spherically symmetric radial Bessel functions and the usual angular spherical functions, the solution is indefinite in the zero. However, in the theory of the envelopes, this problem can be solved by multiplying the envelopes by periodic function. 
11. Conclusion. In this paper, we derive a set of amplitude nonlinear Maxwell equations (NMEs) for nonlinear optical media with and without dispersion of the electric and magnetic susceptibility. We have shown that in cases of linear and circularly polarized components of the electric and magnetic fields, the NMEs reduce to the system of Nonlinear Dirac equations (NDEs). The equations are represented in a spinor form. Using the method of separation of variables, exact vortex solutions for both cases have been obtained. The optical vortex solutions admit classical orbital momentum $l=1$ and classical own momentum $j= \pm 1 / 2$. Here, I would like to explain more about the differences between solutions obtained by separation of variables of the usual linear Dirac equation with potential depending only on " $r$ " (e.g., hydrogen atom) and solutions of the NDEs. In the case of linear Dirac equations with potential, a higher order of radial spherical Bessel functions corresponds to higher-order spherical spinors. On the other hand, for NDEs, a higher number of field components and higher value of localized energy correspond to a higher order of spherical spinors $(l=1,2, \ldots)$. For all radial solutions of NDEs, the zero spherical radial Bessel function $(\sin \alpha r) / r$ is valid. The optical vortices in media without dispersion admit infinite energy integral. The energy integral of the vortex solutions is finite only in some special cases of paramagnetic media with suitable conditions on linear electric and magnet dispersion. Using the Poynting vector for solutions with spin $j=1 / 2$, we find that the energy flow through arbitrary closed surface around our vortex solutions is zero and the localized energy of our solutions circulates in $x, y$ plane. Another important result is that the vortex solutions with spin $j=1 / 2$ without external fields are immovable. The electrical field in the vortices oscillates spherically, while the magnet field oscillates in $z$ direction. The initial investigations on stability of these solutions show the following: while the vortices with spin $j=1 / 2$ are stable, the vortices with opposite spin (charge) $j=-1 / 2$ are not.

All of the above results will be discussed later in relation to nonlinear field theory.

\section{REFERENCES}

[1] S. A. Akhmanov, V. A. Vysloukh, and A. S. Chirkin, Optics of Femtosecond Laser Pulses, Nauka, Moscow, 1988.

[2] D. R. Andersen and L. M. Kovachev, Interaction of coupled-vector optical vortices, J. Opt. Soc. Amer. B Opt. Phys. 19 (2002), no. 3, 376-384.

[3] A. O. Barut, The Schrödinger and the Dirac equation-linear, nonlinear and integrodifferential, Geometrical and Algebraic Aspects of Nonlinear Field Theory (Amalfi, 1988), North-Holland Delta Ser., North-Holland, Amsterdam, 1989, pp. 37-51.

[4] A. B. Blagoeva, S. G. Dinev, A. A. Dreischuh, and A. Naidenov, Light bullets formation in a bulk media, IEEE J. Quantum Electron. 27 (1991), no. 8, 2060-2065.

[5] M. Born and E. Wolf, Principles of Optics, 6th corr. ed., Cambridge University Press, Cambridge, 1998.

[6] R. W. Boyd, Nonlinear Optics, Academic Press, Massachusetts, 1992.

[7] T. Carozzi, R. Karlsson, and J. Bergman, Parameters characterizing electromagnetic wave polarization, Phys. Rev. E (3) 61 (2000), no. 2, 2024-2028.

[8] A. Dacev, Quantum Mechanic, Nauka i Izkustvo, Sofia, 1973.

[9] D. E. Edmundson and R. H. Enns, Fully three-dimensional collisions of bistable light bullets, Opt. Lett. 18 (1993), no. 19, 1609-1611. 
[10] M. D. Feit and J. A. Fleck Jr., Beam nonparaxiality, filament formation, and beam breakup in the self-focusing of optical beams, J. Opt. Soc. Amer. B Opt. Phys. 5 (1988), no. 3, 633-640.

[11] V. I. Fushchich, V. M. Shtelen, and N. I. Serov, Symmetry Analysis and Exact Solutions of Nonlinear Equations of Mathematical Physics, Naukova Dumka, Kiev, 1989.

[12] D. Ivanenko (ed.), Nonlinear Quantum Field Theory, Inostrannaia Literatura, Moscow, 1959.

[13] V. I. Karpman, Nonlinear Waves in Dispersive Media, Izdat. Nauka, Moscow, 1973.

[14] L. D. Landau and E. M. Lifshitz, Electrodynamics of Continuous Media, Nauka, Moscow, 1982.

[15] V. G. Makhankov, Dynamics of classical solitons (in non-integrable systems), Phys. Rep. 35 (1978), no. 1, 1-128.

[16] A. C. Newell and J. V. Moloney, Nonlinear Optics, Advanced Topics in the Interdisciplinary Mathematical Sciences, Advanced Book Program, Addison-Wesley, California, 1992.

[17] J. A. Powell, J. V. Moloney, and A. C. Newell, Beam collapse as an explanation for anomalous ocular damage, J. Opt. Soc. Amer. B Opt. Phys. 10 (1993), no. 7, 1230-1241.

[18] R. Rajaraman, Solitons and Instantons, North-Holland, Amsterdam, 1982.

[19] N. N. Rosanov, V. A. Smirnov, and N. V. Vyssotina, Numerical simulations of interaction of bright spatial solitons in medium with saturable nonlinearity, Chaos Solitons Fractals 4 (1994), 1767-1782.

[20] Y. R. Shen, The Principles of Nonlinear Optics, John Wiley \& Sons, New York, 1984.

[21] Y. Silberberg, Collapse of optical pulses, Opt. Lett. 15 (1990), no. 22, 1282-1284.

[22] G. A. Swartzlander Jr. and C. T. Law, Optical vortex solitons observed in Kerr nonlinear media, Phys. Rev. Lett. 69 (1992), 2503-2506.

Lubomir M. Kovachev: Institute of Electronics, Bulgarian Academy of Sciences, 1784 Sofia, Bulgaria

E-mail address: 1kovach@ie.bas.bg 


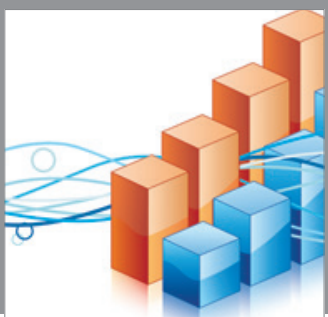

Advances in

Operations Research

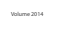

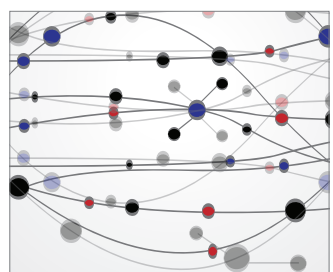

\section{The Scientific} World Journal
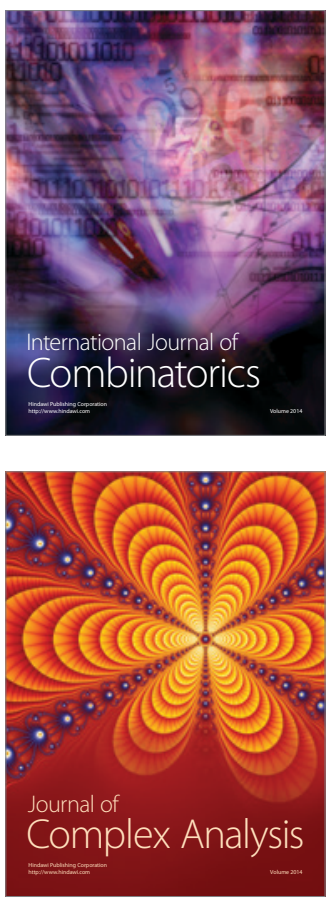

International Journal of

Mathematics and

Mathematical

Sciences
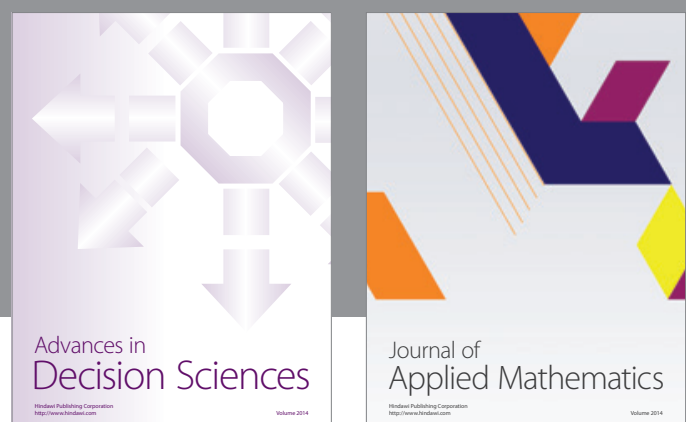

Journal of

Applied Mathematics
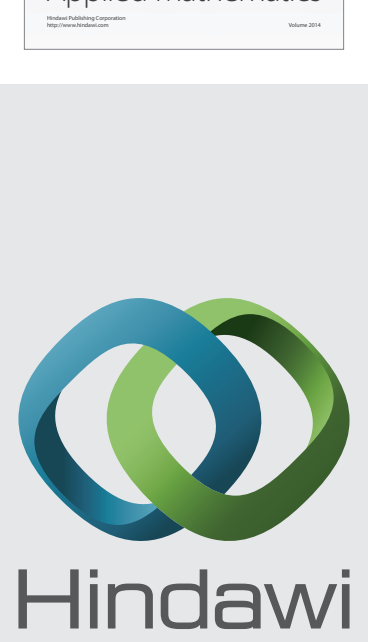

Submit your manuscripts at http://www.hindawi.com
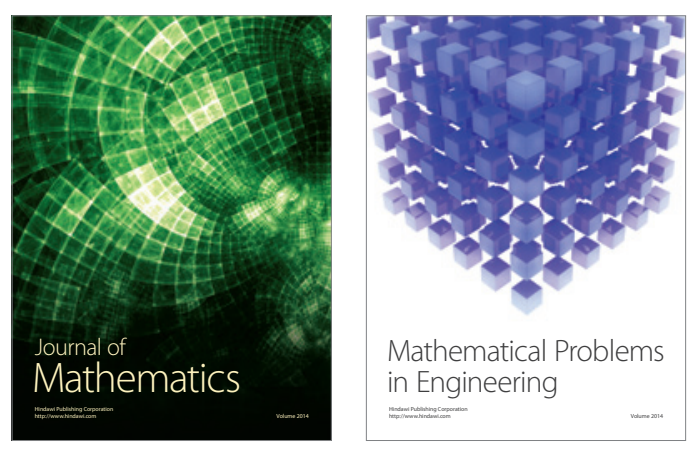

Mathematical Problems in Engineering
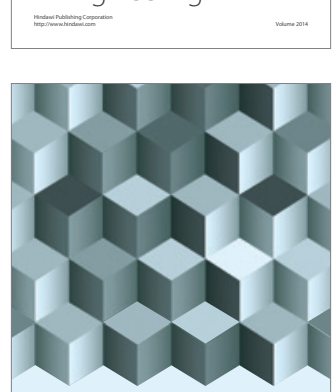

Journal of

Function Spaces
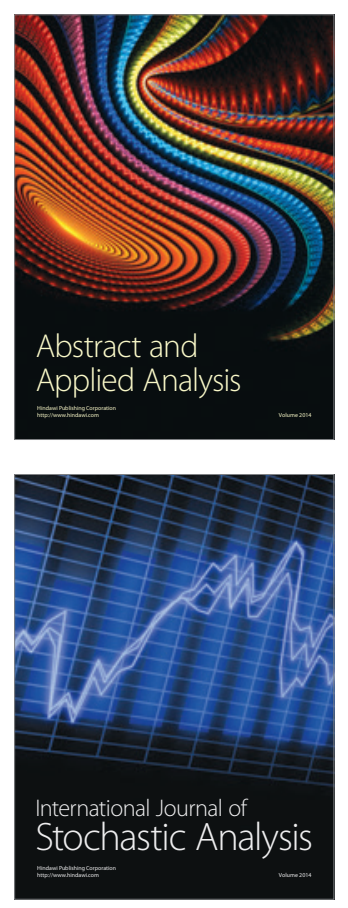

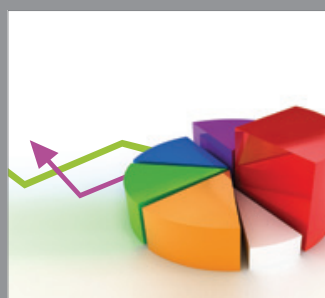

ournal of

Probability and Statistics

Promensencen
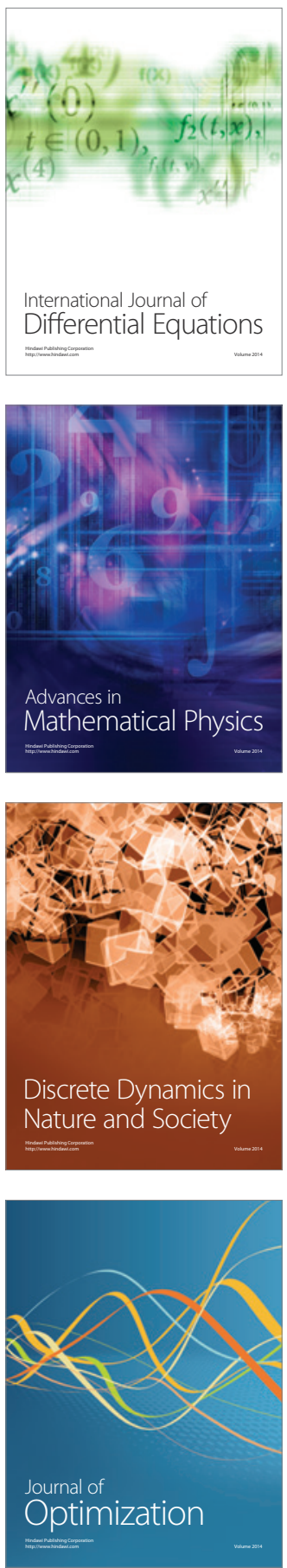